\title{
Philosophical Aspects of Astrobiology Revisited
}

\author{
Rainer E. Zimmermann (D)
}

check for updates

Citation: Zimmermann, R.E.

Philosophical Aspects of

Astrobiology Revisited. Philosophies 2021, 6, 55. https://doi.org/10.3390/ philosophies6030055

Academic Editors:

Marcin J. Schroeder and

Rossella Lupacchini

Received: 26 May 2021

Accepted: 21 June 2021

Published: 2 July 2021

Publisher's Note: MDPI stays neutral with regard to jurisdictional claims in published maps and institutional affiliations.

Copyright: (C) 2021 by the author. Licensee MDPI, Basel, Switzerland. This article is an open access article distributed under the terms and conditions of the Creative Commons Attribution (CC BY) license (https:// creativecommons.org/licenses/by/ $4.0 /)$.
Institut für Design Science München e.V., D-14197 Berlin, Germany; rainer.zimmermann@hm.edu

\begin{abstract}
Given the idea that Life as we know it is nothing but a special form of a generically underlying dynamical structure within the physical Universe, we try to introduce a concept of Life that is not only derived from first principles of fundamental physics, but also metaphysically based on philosophical assumptions about the foundations of the world. After clarifying the terminology somewhat, especially with a view to differentiating reality from modality, we give an example for a mathematical representation of what the substance of reality (in the traditional sense of metaphysics) could actually mean today, discussing twistor theory as an example. We then concentrate on the points of structural emergence by discussing the emergence of dynamical systems and of Life as we know it, respectively. Some further consequences as they relate to meaning are discussed in the end.
\end{abstract}

Keywords: metaphysics; emergence of space and time; twistor theory; dynamical systems; astrobiology

\section{Introduction: Metaphysical Preliminaries}

When we follow the idea to visualize Life as a complex physical system among others supporting the cosmic tendency of maximizing complexity in the sense of Stuart Kauffman ([1], See in particular chapter 3), who introduced this concept within the framework of the Santa Fe school dealing with "self-organized criticality" in nature, then the meaning of Life in particular shows up as this Life's function among the constituents of the Universe, which itself is being visualized as maximal system [2]. Obviously, the latter can be differentiated according to the various research fields dealing with one or the other aspect of the Universe, with physics "at the bottom".

On the other hand, when looking for a definition and explanation of Life and its function in the Universe, the first question is thus: Given insight based in the sciences, why do we deal with metaphysics at all? The reason is simple: While physics, chemistry, and (astro-)biology are obviously located within the sciences as specialized fields of research, even if the latter might turn out to be an interdisciplinary composition of various fields rather than an isolated branch, all of them (by being sciences) are undoubtedly dealing with the observable world that is essentially accessible to human beings by means of their sensory perception in the first place. In other words, astrobiology in particular, making Life topical as visualizing it in terms of the properties of the physical Universe altogether, is trying to grasp a structure of which human beings developing a theory for it are not only a product and a part. More than that, this structure is also endowing human beings with the very instruments that facilitate their reflexive activity (to develop a theory at all). Hence, asking for the emergence, the structure, and the function of Life itself within the Universe-and doing this as a representative of a highly specialized group (of scientists) within humanity on the planet Earth that again can be visualized as a complex structure that is part (actually a special case) of what we deal with (namely, Life), all of that having been produced by this same Universe in the run of unfolding its evolution-we are confronted with a constitutive loop of self-reference that closely connects epistemology to ontology.

This is mainly because the epistemological activity of humans actually is, in principle, their ontology [3]. When performing research, we basically ask for the condition of the possibility to observe something. Explaining in scientific terms thus essentially means reconstructing. While science (physics and the rest) deals with re-constructing processes that 
can be observed, it is philosophical speculation, metaphysics, that deals with re-constructing processes (or conditions of processes) that cannot be observed ([4], See in particular the introduction). However, the latter activity is not redundant, superfluous, or arbitraryinstead, it is necessary in order to compare the possible and the actual. And this is necessary in turn in order to understand the foundations of the phenomena in question.

Assuming then that the physical Universe is all that can be observed, looking for its foundations means to actually "leave" it (in terms of reflexion), because the ground is always external to what is being grounded [5] ${ }^{1}$. This means in particular that principal categories of human thought, such as space, time, matter, and so forth, are concepts utilized in order to describe the observable world. They are thus linguistic fixed points in networks of theories-theories visualized then as sets of propositions that are connected to each other by given rules (of thinking). However, these categories are, in practice, either absent or meaningless when we try to conceptualize the ground of the world. This is the difficulty encountered when dealing with speculation. Nevertheless, there is no arbitrariness involved, because the minimal criterion is that speculation must produce at least all that is already known about the observable world. Hence, both re-construction as well as speculation are formulated in terms of a suitable formal language which utilizes metaphorization in the first place: But the former relies on concrete metaphors while the latter relies on abstract metaphors. At present, a bridge from one to the other can be built in terms of modern mathematics which recently developed as a theory of structure and logic at the same time ([7] ancient series, Cf. also [8]).

Hence, what we will do in this present paper is to offer definitions of the concepts of Life and interpretations of their meanings within the framework of such a formal conceptualization. Nota bene, we will be offering definitions for the concepts of life, which is not the same as offering definitions of life. The problems concerning the many definitions of life and the many attempts to define life in the face of definitions that some researchers already find sufficient are well known; we will not re-plow that field here. Rather we will strive to survey the landscape of definitions underpinning its concepts. This will also touch upon meaning. Asking for the meaning of Life implies a hermeneutic perspective, the point is that hermeneutic in the traditional sense shows up as a logic with limited informationor vice versa: logic shows up as a hermeneutic with a good deal of information. In this sense, for the purpose of describing the complete substratum of the observable world we can utilize the fundamental concepts of matter (energy-mass) and information (entropystructure), respectively ([9], Cf. also [10]). Conceptualizing the ground then means to define the possibility for the condition that determines the emergence of matter and information as seen in terms of a human observer's perspective. [5] And it is the actual degree of complexity that determines the degree of hermeneutic entering the essentially logical interpretation of systems.

We will start then in mathematical terms by investigating what Roger Penrose has called twistor theory ([11], See also [12]). This will serve as a "simulation of accessing the ground". The idea is as follows: Intuitively, the ground of the world is related to, if not identical with, the space of possibilities. It is taken for granted that this space is (much) larger than the space of actualities. This is indeed the reason for having a history within the observable world at all, because there is a possible choice in the first place among those possibilities that are actualized eventually [13]. When thinking of an appropriate mathematical analogue, we would like to visualize the space of possibilities as some kind of manifold of which the space of actualities is a sub-manifold. This can be easily done by selecting a complex space-time analogue: Obviously, a four-dimensional real space-time can be part of (that is embedded in) a four-dimensional complex space-time (which is isomorphic to an eight-dimensional real space-time). Hence, we have to make essential assumptions before starting: First of all, we utilize a manifold model as a first approach, even though we know that a smooth manifold will be an emergent result rather than a fundamental input in the first place, because on the fundamental level (ground2), when conceptualized, space and time (and all the rest) would probably show up as discrete rather 
than continuous concepts. But this does not preclude utilizing a manifold for an abstract space of possibilities.

The second point is that although twistor theory is not yet listed as a standard theory in theoretical physics, for our purpose here it will suffice in order to illustrate the basic idea. (In the meantime, though, the theory has been developed further in different fields [14]. See also $[15,16])$. In brief, twistor theory was originally developed by Roger Penrose in order to describe a conceptual approach to quantum gravity [11]. It is not known to be a fully accurate representation of the latter, but it is also not known to be inaccurate, and it has some interesting mathematical properties. Compared with some alternative approaches to describe quantum gravity, it offers some useful hints as to the initial emergence of structures in both the contexts of classical relativity theory and quantum theory, respectively. Because twistor theory deals with a complex space, we know that the concepts involved are abstract rather than concrete. Nevertheless, we also know that complex space very often serves practical purposes in a purely technical respect, starting with electromagnetic fields. Hence, extrapolated to the problem at hand, as the ground of the world would be some type of allencompassing totality (substance/subject) of what there is (namely, by being possible in one way or the other, or by being actual, respectively), we can utilize the mathematical analogue of a complex space in order to model (if only in an imperfect manner) this totality. This is how the mathematical model can be utilized in order to replace a purely metaphysical model. On the one hand, the disadvantage is that it can be nothing more than an abstract approximation to a classical substance model, because it covers only the formal part of the latter. On the other hand, the advantage is that this type of mathematical meta-phorization is both formally structured and readily comprehensible in terms of what we know from physics. A useful consequence of this advantage is that the formal results can, in principle, connect back to the general theoretical physics framework.

While the mathematical conceptualization turns out to be useful for describing (at least some important aspects of) the ground of the observable world, the next step would be to describe the emergence of dynamic systems that govern the behaviour of the observable world. Especially important for us are those systems that are auto-catalytic, implying their intrinsic non-linearity (including self-interaction properties) and their autonomous evolutionary behaviour. We will find that while the ground carries an implicit self-duality that serves as the origin of a scenario characterized by excited strain which can be visualized itself as the foundation for the observable competitive dynamics of structures, it is this very foundation from which concrete systems can eventually emerge constituting properties that serve as proto-types for those properties we associate with the definition of Life. It is important, however, to keep in mind that emergence does not actually imply a concrete process of production such that the ground of the observable world would actually let this world emanate from itself [17]. Instead, the activity is completely that of the observer and we therefore have to invert the perspective: For human beings, the observable world appears as if it would have been produced by its ground, mainly because the human mode of being depends explicitly on categories such as space and time, prerequisites of motion, categories that we intuitively understand as emergent.

Having conceptualized the ground and the emergent system of systems (which is the physical Universe in fact), we still need to conceptualize the transition from this formal level of dynamics to living systems, or, in short, to Life as we know it. Despite ongoing research, we still do not clearly know how to define Life in this more concrete sense [18]. But essentially, based on the early insight of Eigen and Schuster $[19,20]$, what we can do is to give a conceptualization in terms of evolutionary systems [21] that are emergent, complex, self-organizing, and autonomous in the first place. In other words, it might be easier to understand and recognize Life than to define it, which poses the question of whether we can understand things that we cannot define in the first place. But we will not pursue this further here. 


\section{Substance-Subject in Formal Terms: The Example of Twistor Space}

Classically, the idea of substance metaphysics is that the world as it is being observed (what according to Spinoza's terminology is called modality) is only part of the world as it is (called reality), because humans, due to their characteristic mode of being, have access to no more than two of the infinitely many attributes of substance, namely, matter (res extensa) and mind (res cogitans), respectively. Although today, we would rather visualize mind as a form of matter, and matter itself not only as bulk matter (stuff) of one or the other kind, but also as energy, the essential point has nevertheless remained valid: We still agree that human beings can only gain access to a part of the world, because their means of observation are limited according to their generic capacity of sensory perceptions. And the available projection from reality to modality can still be viewed as a section of the real world as seen in terms of accessible attributes then. However, we would add information to matter now, and hence, our convention here to think of matter (mass-energy) and information (entropy-structure) as attributes of reality in a modern sense. When we turn now to the approach given by Penrose, we recognize a similar argument: This is because the original motivation to develop twistor theory was to look for a structure from which both classical physics (including Einstein's gravity theory) and quantum physics could be derived as a consequence, given the fact that both theories are intrinsically different, but claim equally their validity for a description of the physical Universe [22]. In other words, twistor theory is an attempt to introduce some kind of complete combinatorial hyper-structure of which macroscopic classical physics and microscopic quantum physics are accessible attributes, respectively. Hence, as compared for example to Spinoza, the intention is the same, namely, to derive a differentiated view of the concrete world as an emergent structure out of an abstract ground. This is a formidable task, initially formulated in modern language by Schelling. (For a brief but informative discussion of Schelling's (and Bloch's) work on nature, see also the contribution by Doris Zeilinger in this issue [5].) The difference is only in the epistemological state of the mathematical approach, because twistor theory is given in a formal language backed by empirical results of the sciences involved.

For the sake of brevity, we concentrate here on the essential points of twistor theory only, for further details the interested reader is referred to specialist literature [15] ${ }^{2}$. In principle, twistors are elements of a four-dimensional complex vector space called twistor space II that can be defined as a double fibration over spinor space $S$ and its dual $S^{*}$. Hence, two spinors can be associated with a twistor such that $(\omega, \pi) \in S \otimes S^{*} \approx$ II. In fact, linear transformations on II that conserve exact sequences of the type $\mathrm{O} \rightarrow \mathrm{S} \rightarrow \mathrm{II} \rightarrow \mathrm{S}^{*} \rightarrow \mathrm{O}$ (and their dual) produce the restricted Poincaré group action on Minkowski space. (Here, $\mathrm{O}$ is the trivial group.) If in particular, $\mathrm{x}: \mathrm{S}^{*} \rightarrow \mathrm{II}$ is a section of the fibration, then it defines a motion on $\mathrm{S}^{*}$ in II. However, in order to express realistic physics admitting of space-time curvature in terms of twistors, one has to introduce deformed twistor space DII. Space-time with curvature is called DM. It is called anti-self-dual, if it is conformally transformed and a subset of the complexified future infinity of this transformation. In symbolic terms, this is usually expressed as the relation $C D M^{*} \subset C \Omega^{+}(D M)$, where $C$ points to the complexification and $\Omega$ to the conformal transformation, respectively. It can be shown then that $x \in \mathrm{CDM}^{*}$ as well as $\mathrm{x}^{*} \in \mathrm{CDM}$. In other words, a motion on $\mathrm{S}$, say, defined by $\mathrm{x}$, produces a space-time point on $\mathrm{CDM}^{*}$. The converse is not true. The same can be shown for $\mathrm{x}^{*}$. In fact, spaces $\mathrm{CDM}^{*}$ and CDM are also called Newman's heaven spaces $\mathrm{H}$ and $\mathrm{H}^{*}$, within different approaches. ${ }^{3}$

The close relationship between spinors and twistors secures an important isomorphism between the groups $\mathrm{SL}(2, \mathrm{C})$ and $\mathrm{O}(1,3)$ that mirrors an injective correspondence between tensors and spinors at the same time. Neglecting curvature for a while, one can define a twistor from these basic correspondences alternatively in terms of an equation of the form

$$
\nabla_{\mathrm{A}^{\prime}}{ }^{\left(\mathrm{A}_{\xi}^{\mathrm{B}}\right)}=0
$$


which is solved by

$$
\xi^{\mathrm{A}}=\omega^{\mathrm{A}}-\mathrm{ix} \mathrm{AA}^{\mathrm{AA}} \pi_{\mathrm{A}}{ }^{\prime} .
$$

Hence, twistor space II is the vector space of the solutions of this equation which is thus called twistor equation. As already mentioned above, a pair of spinors can be utilized to define co-ordinates for this four-dimensional complex space. This pair of spinors, written as $\left(\omega^{\mathrm{A}}, \pi_{\mathrm{A}}^{\prime}\right)$, can be identified with a twistor $Z^{\alpha}$. If the conformal group acts on II, then the inner product

$$
\omega^{\mathrm{A}} \pi_{\mathrm{A}}^{*}+\omega^{* \mathrm{~A}^{\prime}} \pi_{\mathrm{A}}^{\prime}
$$

is conserved. Hence, $Z^{\alpha} Z_{\alpha}^{*}=2 \mathrm{~s}$, where $\mathrm{s}$ is the helicity. (This is because a spinor with $2 \mathrm{~s}$ indices represents a physical field with helicity s.) Indeed, visualized this way and for a "flat" space, a twistor corresponds to a pair $\left(\mathrm{p}^{\mathrm{a}}, \mathrm{M}^{\mathrm{ab}}\right)$ expressing the momentum and angular momentum of a particle field of rest mass zero such that $\mathrm{p}_{a} \mathrm{p}^{\mathrm{a}}=0$ and $\mathrm{p}^{0}>0$. (All of this being written in the conventional four-notation of relativity theory.)

A null twistor in particular now represents a null curve in space-time and thus a light ray. If we choose photons with spin and energy and given helicity, what we do is to move in a projection which is called projective twistor space PII consisting of two halves, namely, $\mathrm{PII}^{+}(\mathrm{s}=\mathrm{h})$, and $\mathrm{PII}^{-}(\mathrm{s}=-\mathrm{h})$. By means of defining representations of twistor wave functions, Penrose can utilize this approach in order to give a table of particle fields that can be directly produced from twistor properties with varying helicities. This deals in more detail with contour integrals on holomorphic functions, which we will not discuss here ${ }^{4}$. But the underlying idea should have become clear enough: We have a model here which in principle is able to describe various forms of emergence, of particle fields as well as of space-time points. (Hence, the latter two are less fundamental than the twistor structure itself.) The underlying "attributive motivation" is quite apparent.

It is important to note here once more the important role of what Penrose called spin networks: Because the original idea is to connect complex numbers in quantum mechanics with the structure of space, spin states (of a spin $\frac{1}{2}$ particle say) are especially relevant by being complex linear combinations of two states actually, which we can call "spin up" and "spin down", respectively. This idea underpins the local isomorphism $\mathrm{SU}(2) \rightarrow \mathrm{SO}(3)$, and this isomorphism in turn is what gains importance at the roots of spin networks. Essentially, a spin network can be visualized as a mathematical graph whose nodes (knot points) are entanglement operators and whose edges are labelled by spin numbers (representing the total angular momentum of the knots interpreted as abstract objects of the type $n / 2$ when taking $h=1$ ). The integer $n$ is actually what labels the edge (line segment). In principle, this can be thought of as a network of abstract particles (objects) that exchange spin numbers all the time, in other words, that process spin information. It can be shown then that when modifying line segments in this graph, the probability for the outcome in terms of spin numbers can be related to an angle that defines a spatial orientation (as a relation between spatial directions), provided that the network involved is large enough. This can be understood alternatively as a large superposition of parts of the underlying spin network such that, eventually, a portion of (Euclidean) space emerges from it, similar to a process of condensation (and very similar to the conception involved in what is called "de-coherence" at other occasions).

The first approach offered by Penrose (cf. [11] again) had basically two deficiencies: The angular momentum is non-relativistic on the one hand, and spin and orbital angular momentum are not properly combined, on the other. Hence, the group isomorphism above was modified somehow by incorporating the Poincare group. In fact, the latter was even enlarged further to yield $\mathrm{SU}(2,2) \rightarrow \mathrm{C}(1,3)$ with $\mathrm{C}(1,3)$ being the identity-connected component of the 15-parameter conformal group of (compactified) Minkowski space-time. One consequence is that the spin numbers on the edges of the spin network are elements of the representation of the group SL $(2, C)$ important for all Lorentzian issues in Relativity theory. Without going into too many details, we can state here that in the long run we find 
that a twistor can be visualized as a special spinor for the group $\mathrm{O}(2,4)$ and that the twistor group itself is $\mathrm{SU}(2,2)$ such that $\mathrm{SU}(2,2) \approx \mathrm{O}(2,4) \approx \mathrm{C}(1,3)$.

Hence, twistor theory connects to our current endeavour as follows: For Penrose, twistor theory is primarily a study in complex non-locality on the way to a full model of quantum gravity [30], (p. 424). By virtue of being complex (in mathematical terms), the spaces involved uncover a role of complex geometries within the framework of both classical and quantum physics. This is (mainly) because we know from a long tradition in physics that complex variables can help to find solutions of (real-numbered) problems by means of introducing a kind of diversion technique. This was encountered for the first time in Maxwell's theory of electromagnetic fields. As it turns out, especially for dealing with space-time proper, there is inter alia a close relationship between complex manifolds and the generation of new solutions to Einstein's equation. A recent survey has been presented by Deloshan Nawarajan (Del Rajan now) [31]. The idea traces back at least to the 1976 book by Edward J. Flaherty, who assembles relevant aspects of complex manifold theory and points to an interpretation of complex co-ordinate transformations (important for the generation of new metrics in General Relativity theory) that relates them to imaginary displacements of a complex centre-of-mass line within Newman's heaven space [32], (p. 254). Flaherty also points to the important relation between curves in twistor space and points in heavenly space-times. Because the future null cone at infinity is involved, we are back to the works of Penrose: The formalism named after Newman and Penrose, in whose framework all these aspects are formulated in a straightforward manner is the best illustration for a deep underlying connection relating complex spacetimes, the generation of metrics, twistor theory, as well as heaven theory to each other [32], (pp. 334-344). Although this connection is still far from being understood in a sufficiently clear-cut manner, we can take it as an indication that affirms the original "attributive" motivation mentioned above ${ }^{5}$. At the same time, the categories of space and time are still intrinsically available, but they change their connotation, because on the level of twistor space, there is no manifestation of a similar evolution with an unfolding of processes as we have it on the level of Einsteinian space-times. In other words, relative to our observational standpoint, twistor space (and heaven) is (are) eternal, i.e., space and time as we know them, are absent. The absence of potentially corresponding observations could impact our concepts of Life, depending upon its nature. This presents a caveat for categorization of any Life other than that which we know.

\section{The Emergence of Auto-Catalytic Systems}

One of the oldest problems is that of the initial emergence of an observable world: This is mainly because it is not clear at all why some kind of universally stable (in a sense ideal) ground state would spontaneously convert into states that are far less stable. However, for the world as we observe it within the framework of categories like space and time, it is characteristic state transitions that essentially increase the complexity of the systems involved so that, locally, stability decreases tending towards non-equilibria, while globally, it is instability instead which decreases such that the maximal system tends towards an all-encompassing equilibrium in the long run. We typically attribute this to the laws of thermodynamics. But these are a product of the initial emergence in the first place. This brings us back to the ancient question celebrated in detail by Schelling among other protagonists: Why is there something at all rather than nothing? This very something is obviously constituted in a highly dynamic way. In formal terms, the various processes involved can be traditionally described in terms of differential equations (or "dynamical systems" in the mathematical sense). Indeed, the concept of process itself (or motion as to that) is inseparable from the categories of space and time in particular.

How to achieve insight into an initial dynamical system? Stuart Kauffman bases his concept of "autonomous agent" on the idea of a dynamical origin within fundamental physics leading subsequently through the different levels of increasing structural complexity as we can observe it. Following this idea, we think of agents in the generalized 
sense as self-reproducing, auto-catalytic systems which achieve a new kind of closure in a given space of catalytic and work tasks propagating work out of non-equilibrium states and playing natural games according to the constraints of their environment ${ }^{6}$. In particular, (physical) space is visualized then as being comprised of autocatalytic autonomous Planck scale agents co-evolving with each other serving at the same time as some sort of crystallization of seeds of classicity (in the physical sense). This is an argument that runs roughly parallel to what is usually called "de-coherence" [34]. The co-evolution of agents takes place according to what Kauffman calls the 4th law of thermodynamics: The maximum growth of the adjacent possible in the flow of a non-ergodic Universe maximizes the rate of de-coherence and thus the emergence of classicity. In the long run, there is a hierarchy of such agents depending on the explicit complexity such that human agents in particular (as constituents of social systems) represent a stage of higher complexity as compared to physical, chemical, or biological systems, respectively. (Games of various types of agents are nicely illustrated by Szabó and Fáth [35].) But on the fundamental level of physics, Kauffman mentions the possibility to visualize spin networks themselves as knots acting on knots to create knots in rich coupled cycles not unlike a metabolism. Hence, they (or their constituents) show up as a sort of "fundamental agents". We can suppose from this that the intrinsic self-duality of twistor space (as formal analogue of substance-subject) can be translated as a competitive dynamics of games on the observable level of worldly phenomena. This competitive dynamics can be classified according to its degree of complexity. The substratum of the observable world (matter and information) is thus invariant, while complexity increases according to the laws of thermodynamics. Life thus occupies a specific level of this complexity. As compared to spin networks, the various forms of life belong to an elaborated organization within the given range of a hierarchy of forms that have emerged. Hence, Life occupies a special position within this hierarchy, but it does not transcend the general layout of emergent systems.

If we take Kauffman's viewpoint seriously, then it appears to be straightforward to find the fundamental agents in the loops of loop quantum gravity (and the associated quantum information theory) in the first place: this is because the loops combine in order to form spin networks. In fact, six of them co-operate in order to produce one hexagon of a network. The conceptual reason for this is that the associated entropy satisfies the criterion for a thermodynamic cycle process such that

$$
\frac{1}{4} \mathrm{l}_{\mathrm{p}}^{2} \int \mathrm{d} a \leq 0
$$

where the integral is a closed path integral (loop integral) and $a$ is the surface area generated with respect to one hexagonal fragment of the spin network. ( $l_{P}$ is the Planck length.) By the definition of loops above, we clearly recognize that this procedure is not referring to some physically "vacuous" geometrical meaning, but that, instead, this geometrical picture is physically loaded due to the parallel propagator with its gravitational or curvature connotation, respectively, and the explicit group action involved: Hence, with a loop [36] we mean here a closed curve $\alpha$ such that $T[\alpha]=-\operatorname{tr}\left[U_{\alpha}\right]$, where

$$
\mathrm{U}_{\alpha}\left(\mathrm{s}_{1}, \mathrm{~s}_{2}\right) \sim \mathrm{P} \exp \left\{\int \mathrm{s}_{\mathrm{s} 1} \mathrm{~A}_{\alpha}(\alpha(\mathrm{s})) \mathrm{ds}\right\}
$$

is the parallel propagator of the vector field $\mathrm{A}_{\alpha}$ along $\alpha$ defined by (the $\mathrm{s}_{\mathrm{i}}$ being points of $\alpha$ )

$$
\mathrm{d} / \mathrm{ds} \mathrm{U}_{\alpha}(1, \mathrm{~s})=\mathrm{da}_{\mathrm{i}}(\mathrm{s}) / \mathrm{ds} \mathrm{A}_{\mathrm{i}}(\mathrm{i}(\mathrm{s})) \mathrm{U}_{\alpha}(1, \mathrm{~s}) \text {. }
$$

The SO(3)-field A is here essentially the difference of the SU(2)-spin connection and the extrinsic 3-curvature called real Ashtekar connection:

$$
\mathrm{A}_{\mathrm{i}}^{\mathrm{j}}(\mathrm{x})=\Gamma_{\mathrm{i}}^{\mathrm{j}}(\mathrm{x})-\mathrm{k}_{\mathrm{i}}^{\mathrm{j}}(\mathrm{x}) .
$$


The important result (Rovelli) ([37], see also [38]) is that each spin network state can be decomposed into a finite linear combination of products of loop states. Obviously, this bears a strong resemblance to the Wilson loop representation (hence, we think here of a kind of loop transport according to Kauffman's idea of agents), and is also essentially a Feynman-type integral which gives the probability for a (physical) system to go from one state to another:

$$
<\mathrm{x} 2, \mathrm{t} 2 \mid \mathrm{x} 1, \mathrm{t} 1>=\int_{\mathrm{x} 1}^{\mathrm{xN}} \mathrm{D}(\mathrm{x}(\mathrm{t})) \exp \mathrm{i} / \mathrm{hS},
$$

where $S$ is he action of the form

$$
S:=\int{ }^{t} 21 d t L\left(x, x^{\prime}\right)
$$

Here, $\mathrm{L}$ is the Lagrangian. The probability is the above expression squared. This is equivalent to the Schrödinger picture of quantum physics on the one hand and a model for quantum computation on the other. As can be shown, spin networks and spin foams, respectively, can be visualized as Feynman integrals of this type such that the formal Feynman perturbation series of the partition function

$$
\mathrm{Z}=\int \mathrm{D} \varphi \exp (-\mathrm{S}[\varphi])
$$

is given by

$$
\mathrm{Z}=\Sigma_{\mathrm{J}} \mathrm{N}(\mathrm{J}) \Sigma_{\mathrm{e}} \mathrm{II}_{\mathrm{f} \in \mathrm{J}} \operatorname{dim} \mathrm{a}_{\mathrm{f}} \mathrm{II}_{\mathrm{V}} \mathrm{A}_{\mathrm{v}}(\mathrm{e}),
$$

where $\mathrm{J}$ is a 2-complex, and the vertices, edges, and faces are labelled accordingly. It is $\mathrm{N}(\mathrm{J})$ the number of vertices of J divided by the number of symmetries of J [37]. (In fact, we deal here with a quantum computation that can be utilized in a straightforward manner to serve the quantization of space and time.)

There is a number of important cross-relationships which connect the notion of loops with the notion of knots: Louis Kauffman's bracket algebra (the boundary algebra of containers and extainers) turns out to be the precursor of the Temperley-Lieb algebra important in order to construct representations of the Artin braid group related to the Jones polynomial in the theory of knot invariants ([39], See also [40] ancient series, and [41]). As the elementary bracket algebra is to biologic what Boolean logic is to classical logic, this has important epistemological consequences. On the other hand, the Jones polynomial can itself be visualized in terms of quantum computers, because a similar partition function of type $\mathrm{Z}_{\mathrm{K}}=<\operatorname{cup}|\mathrm{M}|$ cap $>$ with creation and annihilation operations, respectively,

$$
\begin{gathered}
\text { cup: }=|\mathrm{a}\rangle: \mathrm{C} \rightarrow \mathrm{V} \otimes \mathrm{V}, \\
\text { cap: }=\langle\mathrm{b}|: \mathrm{V} \otimes \mathrm{V} \rightarrow \mathrm{C},
\end{gathered}
$$

$\mathrm{M}$ being the braiding, and $\langle\mathrm{K}\rangle:=\Sigma_{\sigma}<\mathrm{K}|\sigma\rangle \mathrm{d}^{|| \sigma||}$, can be related to the process of quantum computation (similar to the spin network formalism itself). As spin networks are nothing but graphs, the agency in question here is motion on graphs or percolation of energy and information in networks such that phase transitions can be represented in terms of an appropriate cluster formation of connected components. This is what points to a close relationship to cellular automata utilized for the simulation of evolutionary processes (cf. Conway's game of life or Wolfram's approach). Stuart Kauffman has associated this with the emergence of collectively autocatalytic sets of polymers, and in fact with the onset of forming classicity with regards to physics. It is straightforward (in epistemic terms) to generalize this (with a view to higher-order agents) to chemical, biological, and other systems.

But there is still another point to this set of observations: In the approach of Barrett and Crane [42,43], the idea is to generalize topological state sum models in passing from three to four dimensions by replacing the characteristic $\mathrm{SO}(3)$ group with $\mathrm{SO}(4)$, or its appropriate spin covering, $\mathrm{SU}(2) \times \mathrm{SU}(2)$, respectively. The concept of spin networks is 
also generalized then, by introducing graphs with edges labelled by non-negative real numbers (called "relativistic spin networks"). Applying this kind of "spin foam" model to Lorentzian state sums demonstrates their finiteness in turn implying a number of choices made from physical and/or geometrical arguments. The important aspect of this is its relation to the group $\mathrm{SL}(2, \mathrm{C})$ mentioned above, because this is the double cover of $\mathrm{SO}(3,1)$ and the complexification of $\mathrm{SU}(2)$ which in turn is the double cover of $\mathrm{SO}(3)$. On the other hand, SL $(2, C)$ is the group of linear transformations of $C^{2}$ that preserve the volume form. Thanks to an e-mail crash course on these matters referring to the Barrett-Crane model and made available online by John Baez and Dan Christensen [42,43], it is easy to understand that constructions in the sense of Barrett-Crane turn out to be invariant under SL(2,C). In other words, we essentially deal with states in $C^{2}$ which are spinors. And it is from quantum theory and special relativity, especially by the important work of Penrose discussed above, that we know about their relevance. On the other hand, as Baez notes, a state in $\mathrm{C}^{2}$ can also be called a qubit. So "[w]hat we [a]re really doing, from the latter viewpoint, is writing down 'quantum logic gates' which manipulate qubits in an SU(2)-invariant [in fact, SL(2,C)invariant] way. We [a]re seeing how to build little Lorentz-invariant quantum computers. From this viewpoint, what the Barrett-Crane model does is to build a theory of quantum gravity out of these little Planck-scale quantum computers" [44], (p. 42).

This allows us to visualize spin networks as a kind of "boundary layer" spread out between the physical world and its foundation (traditionally called a subject or natura naturans) that shows up as a "shift of quantum computing" processing the fundamental information necessary for representing the transition from foundation to world (usually taken for a motion which actually produces a world out of its foundation). Utilizing what I have once called the "skeleton-of-the-universe view", the idea would be to insert various steps of a hierarchy of complexity in the overall functor diagram from topological quantum field theory [45]:

$$
\begin{array}{ll}
\mathrm{nCob} \rightarrow & \text { Hilb } \\
\uparrow \mathrm{em} & \uparrow \downarrow \text { id } \\
\text { SpinF } \rightarrow & \text { Hilb }
\end{array}
$$

This diagram is commutative, if an adequate emergence (em) mapping is being defined. Here, SpinF is the category of spin foams, and nCob is the category of n-dimensional cobordisms. (We can safely set $n=4$.) As I have shown at other occasions, a suitable choice of the von Neumann entropy of the form $S(\rho)=\operatorname{tr}(\rho \ln \rho)$, where $\rho$ is a suitable density matrix, fulfils Stuart Kauffman's condition for an autonomous agent such that we have for the loop integral over $\gamma: \int_{\gamma} \mathrm{d} S(\Omega) \geq 0$. It is especially the Braunstein-GhoshSeverini (BGS) entropy in fact which is relevant here: This refers essentially to a quantum field theory on a space $\Omega \subseteq \Sigma$ with $\mathrm{H}_{\Sigma}=\mathrm{H}_{\Omega} \otimes \mathrm{H}_{\Omega}{ }^{\mathrm{C}}$ as adequate tensor product of the associated Hilbert spaces such that $\rho_{\Omega}=\operatorname{tr}_{H(\Omega, C)}|\psi\rangle<\psi \mid$. In particular, for the loops of this theory, the appropriate Hilbert spaces are defined by the cyclic functions of an $\mathrm{SU}(2)$ connection A. Hence: $\left\{\psi ; \psi(A)=f\left(U\left(A, \gamma_{1}\right)[\ldots] \mathrm{U}\left(\mathrm{A}, \gamma_{\mathrm{L}}\right)\right)\right\}$, where $\mid \psi>$ is a spin network state. The density matrix of the underlying graph $\Gamma$ turns out to be the Laplacian matrix $\mathrm{L}(\Gamma)$ (essentially the difference between degree matrix and adjacency matrix) divided by the degree-sum of such a graph. In a sense, the "skeleton-of-the-universe view" can be thus visualized as a generalized kind of an algorithmic approach such that the physical phenomena of the universe (i.e., of the observable physical world) emerge as a result of algorithmic procedures that are coded into a procedural space [46] that is logically prior to the action of fundamental quantum computation.

As far as graphs and networks in the physical Universe are concerned, the emergence of dynamical systems shows up as a generic consequence of the emergence of space and time. Nevertheless, auto-catalytic systems, which are topical in biological evolution, are usually represented in terms of classical differential equations. Hence, we also have to ask why this type of representation emerges. As Kaneko Kunihiko showed some time ago [47], it is because networks show up as the dynamical core of systems, that their underlying dynamics implies a representation in terms of differential equations in a straightforward 
manner. On the one hand, this takes care of a stage of low-scale change of existing features in systems, while on the other hand, the stage of the emergence of novel features entailing a mechanism for how complex higher-level behaviour emerges from low-level interactions point to strongly non-linear interactions among agents. I have included these aspects in my own definition of systems [2], (p. 27), the difference being that Kunihiko deals with stochastic differential equations from the outset. The idea is that the non-linear interactions involved imply a chaotic behaviour causing a kind of sublation by which lower-level conflicts are resolved. Hence, chaos shows up as the most universal mechanism to create complexity serving at the same time as a source of diversity. In other words, identical elements of the system differentiate through chaotic dynamics. The maintenance of the achieved diversity is what Kunihiko calls homeochaos. We note the discrete rather than continuous starting point for a discussion of these phenomena. Consequently, the basic aspects start with cellular automata (especially in the sense of the Santa Fe school) such that networks of chaotic elements are globally coupled to each other. Nevertheless, the discrete version of the time evolution of labelled elements of systems, can be comparatively easily expressed, by writing

$$
x_{n+1}(i)=(1-\varepsilon) f\left(x_{n}(i)\right)+\varepsilon / N \sum_{j=1}^{N} f\left(x_{n}(j)\right),
$$

where we have $n$ discrete time steps, $\mathrm{i}$ denotes the index of the respective element $(\mathrm{i}=1 \ldots$ $\mathrm{N}$ ), $\varepsilon$ is a suitable coupling (if $\varepsilon=0$, then the motion is chaotic, with $a$ large enough), and $\mathrm{f}(\mathrm{x})=1-a \mathrm{x}^{2}$.

Inspired by the theory of neural nets, we can find then that parallel non-linear transformations show up that possess feedback loops generated from the mean field. We can thus differentiate a coherent phase, an ordered phase, a partially ordered phase, and a turbulent phase, respectively, within the evolutionary processes involved. Hence, we observe the emergence of a generic dynamics. In principle, the behaviour is governed then by the competition among attractors of the system. What can be especially called open chaos [47], (p. 175) can be discussed in terms of an ongoing expansion of the associated phase space exhibiting Baker-type transformations. (It is interesting to note the relationship to the earlier works of Prigogine here.) We can also find similar approaches under various perspectives in other fields of research ${ }^{7}$. More recently, Kauffman has collected all the various aspects in order to present a concise approach to his concept of "constraint closure" [56]. Referring to the work of Mael Montévil and Matteo Mossio [57], he starts from the connectivity of random graphs that eventually leads to dynamical structures and metabolisms [56], (p. 40 sqq.). The results fit nicely the original ideas of the seminal paper on the replication of polymers which at the time was preparing further work about the stochastic onset of network growth [58].

What we find in the end is a kind of formal narrative ${ }^{8}$ that starts with the formation of auto-catalytic (strictly self-organizing) network structures from which discrete dynamical systems emerge that can be expressed in turn by continuous differential equations, once the appropriate limit is chosen. The physical beginning of this procedure can be located in the initial co-operation of loops when forming their spin network hexagons that lie at the bottom of space and time proper. But in order to find a reason for the explicit necessity of this dynamical onset, we have to go back to the metaphysical ground mentioned in the beginning. When trying to find a formalized parallel to this ground by means of choosing the example of twistor theory, as we have done above, we can argue as follows: It is the intrinsic self-duality of the spin space construction of the form $S \otimes S^{*}$ within the framework of this theory that creates an immanent tension which serves as the impulse for the onset of differentiation. Compare this with the theory of de-coherence: At a given order of magnitude (in the nanometer range), the quantum coherence of entangled states is spontaneously broken (in the eyes of the theory, that is) such that a classical world is created. Initial chaos can be compared here with original quantum coherence. This situation is very similar to what we have discussed here: Originally, twistor space shows up as a kind of procedural space containing intrinsic tension. In other words, twistor space 
is to the observable world what a computer program is to what is being processed and observable on the computer screen. The difference may be that a strict algorithm might be absent. (This is still being discussed.) Once space and time have formed and the dynamics is triggered, evolution accelerates due to the underlying thermodynamics that provides the laws for a necessary strife for diversity—especially according to Kauffman's fourth law. Remember, however, that this forming, triggering, and accelerating is simply in the picture of the theory developed by those who conceptualize what they observe. (In fact, this comes nearest to the perspective of substance metaphysics from Spinoza to Schelling and further on.) The relevance of this to Life in particular is that it emerges thus spontaneously from a whole bundle of properties and conditions that have been acquired by systems due to the processes involved while translating the implicit structural imprint on procedural space into explicit dynamical phenomena on concrete space-time. Hence, essentially, Life is nothing but an epiphenomenon, perhaps necessary, but in any case contingent. It serves the diversity of what there is.

\section{Life as We Know It}

Departing from the conceptual and returning to the observational, the question remains whether the dynamical structures discussed so far can already be labelled as representing Life. Obviously, in epistemological terms, the main problem is that of animism, because, if loops are forms of Life, then the Universe altogether can be visualized as one organism. (This is in fact not a new idea at all, see e.g., more recently [59].) But if this is the case, we lack an accuracy of definition for the concept of Life, because it would not have any selective value: if everything is Life, nothing is. On the other hand, it is not satisfactory to let Life start with RNA and DNA structures, because it is not the "stuff" that is decisive, but, according to the propositions outlined above, the underlying dynamics of the interactions taking place. By that measure, exactly this "competitive strife for dynamics" founds the necessity of Life and the acceleration of its evolution.

Some time ago, Lee Smolin offered a suitable definition for Life: He calls a living system one that is a self-organized non-equilibrium system such that its processes are governed by a program which is stored symbolically, and it can reproduce itself, including the program [60], (p. 156). While Smolin himself agrees that the first half of the definition would be true for the whole Universe, he disputes this for the second half, because he thinks that laws of nature could not be visualized as programs in the definition's sense [60], (p. 157). Without getting into the question of whether astrobiologists concur on Smolin's definition, his proposition is generally somewhat difficult to understand, because thinking of "laws of nature" as rules that are imposed upon processes by defining a conceptual framework (under the perspective of human observers), implies their encoding in lines of a program text in the first place. This is mainly because the mathematical formulation itself is essentially nothing but such an encoding after all ${ }^{9}$. The search for natural laws is a search for the language in which they are written. (The list of lines giving mathematical formulae for a problem in question and the list of lines giving a digital program for this same problem are isomorphic.)

Indeed, Smolin himself points to the important relevance of interstellar matter in the becoming of material forms within cosmic evolution [60], (p. 123) ${ }^{10}$. Consequently, this is the generic starting point for a possible continuation of such an approach into the detailed micro-structure of material forms. We see from the example given by the Aquarius project mentioned in note 8 that the evolution of interstellar matter defines a motion which can be taken as ground for the further onset of the formation of structure through a generalization of reaction networks of all kinds. (And obviously, the Aquarius project is simply based on a digital program.)

These aspects highlight the conceptual problem we always encounter when dealing with the problems germane to this present paper: Traditionally, mathematical modelling utilizes local scenarios which are linked by means of essentially undefined singular points where the process leaps from one scenario to the next. Hence, we usually deal with 
questions concerning transitions of various kinds, for example, from radiation to matter, from inorganic to organic matter, from organic matter to living matter, from living systems to thinking systems. But details about how these transitions took place in the historical sequence of events that started on the early Earth ultimately gave rise to humans are unknown. An alternative to the transitions puzzle would be a programmed algorithm that starts from a given moment and unfolds structural content in series by itself. Computer simulation can achieve that and in doing so contributes to the understanding of selforganization. The singular points of the onset of transitions then emerge themselves from within the process.

In a contribution to this issue, Martin and his co-workers provide a short list of alternative narratives for the origin of Life following various possible pathways of evolution [63] ${ }^{11}$. Most of these points are compatible with the view pursued here. This is also the case for what Dirk Schulze-Makuch and Louis N. Irwin discuss in their book when for a suitable definition of Life they collect a number of properties common to living beings [64], (p. 7 sqq., ch. 2). But when they also collect exceptions to their list of properties, then we notice that these do not necessarily contradict the principles mentioned above. In Smolin's definition, the phrase "governed by a program" hides all that we usually connect with the intentional participation of agents in games up to explicitly Darwinian behaviour. If we apply this definition and also assume the existence of the postulated program, then the definition I have given for systems in [2], (p. 27) can be readily utilized. However, in that case the definition is very general, and the Universe shows up as a maximal system that consists of a multitude of sub-systems, essentially a system of systems [65], (p. 130).

Another unclear point is the distinction proposed by Schulze-Makuch and Irwin as to the condition of being alive on the one hand and properties of a living system on the other [64], (p. 14). Despite the argument that this distinction would be useful when looking for traces of Life on exo-planets, while at the same time many individual samples of Life forms would not permanently rely on their properties at all, definitions should concentrate on the general case in principle. Hence, the colloquialism often misattributed as a quotation from the Star Trek I movie series (It is life, Jim, but not as we know it) gains its philosophical impact: It is the living system we know whose details are listed in the definition, independent of whether we are able to observe an individual sample that fits this definition. So that in the end, we have to refer to a convention when specifying whether we talk about Life or not. In the definition of a system given above, the individual components (parts) are the agents whose network defines the dynamical core of the system. But if we change the level of observation, these agents are systems themselves (namely, on a subordinate level).

Hence, while we would find a distinction between the state of being alive and given properties of a living system to be not actually necessary (if arguing on the general level), what we would like to differentiate instead is the concept of system (in the sense of the above definition) and living system, respectively: The idea is that the quality of the agents decides about the label. As it is unlikely that Life (as we know it) develops beneath a minimal threshold of molecular organization, it might be justifiable (if not completely satisfactory) to identify living systems with agents whose structure is determined by RNA-like or DNAlike molecules. Though this argument looks somewhat circular, it is nevertheless legitimate within the framework of a "life-as-we-know-it" guideline. Reading the definitions strictly means that debated questions such as whether viruses are living systems or not can be decided to the negative, because a virus cannot reproduce itself. A virus of one type represents a system however that interacts dynamically with its environment. And the host organism is part of this environment. (Recall, however, that humans require a gut microbiome to survive. In that sense, humans cannot reproduce without help from other organisms either, hence would also fall short of being alive for the same reason as viruses under some definitions.) Reproduction alone is not a sufficient criterion, though, because one could argue that populations of elementary particles, atoms or molecules, can also reproduce, if only based on strict boundary conditions steered by the laws of nature. On 
the other hand, spontaneous reproduction would also not be decisive, because it is not quite clear whether one would visualize animals as spontaneously reproducing (if, say, depending on a fixed season or a 17-year cycle like locust). Finally, a criterion like Darwinian reproduction might look more to the point, although such criteria tend to be tailored towards including RNA-like or DNA-like stuff (molecules), which few specialists and even fewer generalists would understand or designate as being alive, hence constituting a form of Life.

In other words, the concept of system can be grounded in the origin of the Universe. The characteristic structure of its dynamical core, i.e., its network structure, is universal itself and governs the onset of more structure, including what we call "stuff" in the long run. As far as Life is concerned, the underlying dynamics (triggering the emergence of competitive, complex agent-directed structures as sub-systems within the maximal system) is the invariant that steers the further evolution within the framework of space and time, of matter (as energy-mass) and information (as entropy-structure). In a sense, we could call all these earlier systems proto-life. The same would be actually true for the concept of subjectivity (very much in the sense of the philosopher Ernst Bloch). Speaking of a "co-operation" of loops, in order to form a hexagon of the spin network implies a kind of subjective interaction which is, however, pre-established according to the fundamental rules of physics. But "co-operation" also entails the recognition of what is being preferred and an advantage rather than discarded and a disadvantage (pointing to an obvious though rudimentary form of awareness, in fact). Obviously, this is not actually reflexion as we understand it, but it is on the way towards reflexion. Such a result is however nothing but trivial after all, because if we assume a hierarchy of stages of evolution (as we have done here throughout), then the preceding stage is comprised of potential that is the condition for the following stage. Hence, the former is the proto-stage with respect to the latter. As such, much more advanced Life forms might designate humans as proto-life for lack of certain attributes.

While the biological stage follows the chemical and the physical, respectively, it is itself the precursor of the reflexive stage of consciousness. (And generically, research on Life in the Universe implies also research on Intelligent Life.) Hence, we have a kind of hierarchy that is starting with Proto-Life, passing through Life up to Intelligent Lifewithout stopping then. There is no sufficient reason for expecting that evolution would come to an end once Intelligent Life has emerged. Again, Penrose (together with Stuart Hameroff) has published on this problem of emergent consciousness based on a general perspective given by established physics [66 ${ }^{12}$.

Although the approach of Penrose and Hameroff is essentially a quantum theory of consciousness and looks very impressive, to say the least, I cannot actually befriend myself with the idea of a consciousness that is primarily a quantum entity. This is mainly because I prefer the more conventional idea instead that consciousness is a classical entity emergent with respect to the quantum level. This viewpoint is strengthened somewhat by the fact that the categories of human reflexion, obviously a product of consciousness after all, such as space, time, material objects, and so forth, emerge above a given critical threshold in the order of magnitude rather than prevailing from the beginning on through all orders of magnitude. And this emergence depends on the state of observation (of the human observer), as de-coherence theory has been able to show. This does not, however, contradict the justifiable assumption that quantum physics is at the bottom of physics altogether ${ }^{13}$.

It is interesting to note that there is another approach making hierarchically ordered steps of evolution topical again that is mainly compatible with what we have been discussing here [69]. This approach discusses cosmological evolution in terms of a history of the unfolding of consciousness only, starting with entanglement and ending (for the time being) in self-reflexion. Probably, it is useful to visualize this evolution alternatively as one of Life running through various developmental stages. Self-reflexive consciousness would be then a highly organized state of Life, but it would not necessarily end with it. Instead, evolution could be open towards yet unobserved forms of Life, with attributes 
that might not fall under definitions we distill from Earth's sample, both at the simple and the complex end of the spectrum.

\section{Conclusions: Once More the Meaning of Life}

When we collect now our results so far, what we can do is visualize the respective sections as (1) a preliminary clarification of the philosophical terminology derived from the line of thought as characterized by the philosophies leading up to Schelling's approach and further on (towards Ernst Bloch e.g.)); (2) a representation of a formal analogue to substance; (3) a representation of a formal analogue to subject, understood as unified primordial stuff; and (4) a representation of phenomenology in the strict sense. What we find is the following:

When presupposing that the laws of physics (of physical nature) are an emergent property of the Universe that is emerging together with the latter itself, and taking into account that this emergence looks $a$ s if it would be a sort of production of something out of nothingness when viewed in terms of the human perspective, then we have to conclude that the observable properties of the Universe are pre-determined by the Universe's ground in the first place. Re-constructing the history of this Universe would then be seen under a given perspective that lets the observable structures become classified according to a progressive series of stages leading through phases of Proto-Life, Life, and Intelligent Life, with some open ending. There are several possibilities to rephrase this situation: We can speak either in terms of the traditional division of research fields (physics, chemistry, biology, and so forth) or in terms of Life forms as mentioned above. Note that the transition from one stage to the other is at present always poorly understood and for that reason somewhat ill-defined (or vice versa). We could also converse in terms of systems instead, of which living systems and thinking systems are special and highly complex cases. In this case, the original criteria derived from the first definition of systems (as discussed above) deal with the inherent structural stability of the dynamical core. In other words, complex sub-systems of systems have to interact among each other such that their various functions can be permanently harmonized into a self-organizing whole which is defining the system altogether. In the maximal case this would be the Universe. But in most cases, we deal with its sub-systems on a much smaller scale. However, the dominant rules stay the same: Within the framework of balance equations and conservation theorems (as to energy, mass, momentum, and so forth), and according to the laws of thermodynamics in general, interactions among systems and their components are permanently competitive and even contradictory in principle. Sufficient stability can only be achieved if the processes involved remain per force or sui generis within a given pre-defined range of their interaction space [2], (p. 107 sqq.) [70].

Hence, it is very much the function expressed by the actions of a given sub-system that contributes to the stability range in question. In this sense, structure and function are complementary to each other. When referring to an ancient definition given by Wittgenstein [71], then-in the wording of Seth Lloyd- "the meaning of a piece of information is to be found in the action this information provokes" [72], (p. 26). Taking this view of meaning to the relationship between structure and function and applying it to Life, then consequently, the latter shows up as a bundle of functions that improves the original (non-living) system's strife for dynamical stability [48]. The idea is that at the same time this is also Life's meaning after all. The underlying strife for accelerating dynamics emerging out of the world's ground because of this very ground's intrinsic tension represented by its self-duality can only be sufficiently supported if the complexity of the systems involved is permanently increasing according to the given laws. Hence, a function that increases complexity contributes to this overall objective [5,73].

To shortly summarize ${ }^{14}$ : We have noticed that, by asking for the emergence, the structure, and the function of Life (the latter deciding about its meaning after all) within the Universe, we are confronted with a constitutive loop of self-reference that closely connects epistemology to ontology. When performing research, we basically ask for the condition 
of the possibility to observe something. Explaining in scientific terms thus essentially means re-constructing. While science deals with re-constructing processes that can be observed, it is philosophical speculation, metaphysics, that deals with re-constructing processes (or conditions of processes) that cannot be observed. Assuming then that the physical Universe is all that can be observed, looking for its foundations means to actually "leave" it (in terms of reflexion), because the ground is always external to what is being grounded. Although today, we would rather visualize mind as a form of matter, and matter itself not only as bulk matter (stuff) of one or the other kind, but also as energy, the essential point of traditional metaphysics has nevertheless remained valid: We still agree that human beings can only gain access to a part of the world, because their means of observation are limited according to their generic capacity of sensory perceptions. This has obvious consequences for the theories describing the world. For the world as we observe it within the framework of categories like space and time, it is characteristic state transitions that essentially increase the complexity of the systems involved so that, locally, stability decreases tending towards non-equilibria, while globally, it is instability instead which decreases such that the maximal system tends towards an all-encompassing equilibrium in the long run. We typically attribute this to the laws of thermodynamics. The latter form the arena for the dynamics of the world that is emergent with respect to the metaphysical ground. In particular, (physical) space can be visualized then as being comprised of autocatalytic autonomous Planck scale agents co-evolving with each other serving at the same time as some sort of crystallization of seeds of classicity. In a sense, this "skeleton-of-the-universe view" can be thus understood as a generalized kind of an algorithmic approach such that the physical phenomena of the universe (i.e., of the observable physical world) emerge as a result of algorithmic procedures that are coded into a procedural space which is logically prior to the action of fundamental quantum computation. As to consciousness itself, the conventional idea that it is likewise a classical entity emergent with respect to the quantum level appears to be convincing. This viewpoint is strengthened somewhat by the fact that the categories of human reflexion, obviously a product of consciousness after all, such as space, time, material objects and so forth, emerge above a given critical threshold in the order of magnitude rather than prevailing from the beginning on through all orders of magnitude. And this emergence depends on the state of observation (of the human observer), as de-coherence theory has been able to show. This does not however contradict the justifiable assumption that quantum physics is at the bottom of physics altogether. As to the definition of Life then, this implies that its dynamical conditions (based on a network of interacting agents) are coded into the micro-conditions of the observable world as a consequence of its metaphysical ground, but that its specific properties depend on the co-evolution of the chemical material available in terms of the local distribution of matter and information as it can be conceptualized following the results of modern cosmology.

Funding: This research was funded by the Institute for Design Science Muenchen e.V., Germany, by means of a long-term sponsorship within its project group "theoretical foundations" (www. designscience.de (accessed on 24 June 2021)).

Acknowledgments: I owe my particular thanks to Roger Penrose whose permanent flow of illuminating insight was an encouraging inspiration for me since I first listened to his talks when I was a student in London in the years 1973-1974. I also thank Clive W. Kilmister (formerly King's College London) and Thomas W. B. Kibble (formerly Imperial College, London) for their kind and helpful co-operation and advice. Unfortunately, they are not among us anymore. I thank Basil Hiley for giving me the occasion to talk at Birkbeck College London about my ideas on the fundamental aspects of physics during my sabbatical at Clare Hall, Cambridge, in the years 1999-2000. And last but not least I have to acknowledge the support by Louis Kauffman (University of Illinois at Chicago) as a permanent, reliable as well as kind source of inspiration through the years.

Conflicts of Interest: The author declares no conflict of interest. 


\section{Notes}

Traditionally, in metaphysics, the foundations of something are called its ground. We follow here the line of Schelling for whom ground is to what it grounds, non-being. Note that this is a direct reference to the Greek (mostly Aristotelian) tradition, with an intrinsic double meaning: ground1 as what cannot be observed, but is basically underlying this which can be observed = substance (oú $\alpha$ ) - vs. ground2 as what can be observed in principle (but not yet), but is basically underlying this which can be observed

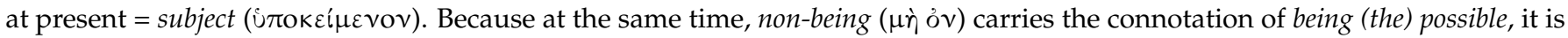
a fundamental demarcation against what is impossible or nothingness (oúk ỏv). Both of these states of being are thus included in the substance, but not in the subject. Note also that Greek concepts of philosophy frequently come from the field of judiciary. Hence, in general, their meaning is difficult to obtain, because of their multiple connotations. While "subject" refers mainly to what "is underlying" something, "substance" can mean in turn "essence", "reality", but also "ability" and "property". Even worse, this European style of "substantivating" a verb (making it to a noun) obscures the fact that originally, we should speak of "substance-ness" or "subject-ness". This somewhat complicates the applications. For more details see Rainer E. Zimmermann: Nothingness as Ground and Nothing but Ground. xenomoi, Berlin, 2014. See also [6]. Among the many books and papers on this topic, very helpful are [23]; as well as [24]. The most recent contributions are [25,26]. I follow here the outline given in [27], notably chapter 3, and [28]. See also [29], notably the appendix, 183 sqq.

See in more detail Zimmermann, R. E. Rem Gerere. Op. cit. [29].

In fact, the oldest example for such an underlying motivation can be found in the early development of Maxwell's theory which represents the simplest theory of unified fields. Essentially, electric and magnetic fields show up here as attributes of a fundamental field called electromagnetic which can be associated with a formal concept of substance. We can notice here an implicit influence of Schelling onto Maxwell (probably mediated by Faraday). For more details on the onto-epistemic consequences of this see [33], sqq. I am referring here to an earlier manuscript version (sfi working paper 96-08-072) of Kauffman's book "Investigations", quoted above [1]. Cf. the address: Available online: https://www.santafe.edu/research/results/working-papers/investigations (accessed on 23 February 2021). In the actual book version, autonomous agents are defined as self-reproducing systems which can at least perform one thermodynamic work cycle.

7 A systematic review can be found in [48]. But see also [49]. On the aspect of small worlds see in particular [50]. More recent works on neural nets are [51-53]. A different, but very promising approach is also given in a large series of papers that started with [54]. This group should be singled out for further work. Finally, as standard work, [55]. The best illumination of formal narratives is by means of computer simulations of complex processes. The Aquarius project e.g. can serve as a useful illustration: Starting the algorithm lets the Universe as we know it presently unfold. Cf. Available online: https: / wwwmpa.mpa-garching.mpg.de/aquarius / (accessed on 13 March 2021). In principle, what we describe in this paper as a formal narrative would be the continuation of the Aquarius simulation project into the detailed micro-structures of the Universe including individual planetary eco-systems.

9 It is useful to compare this with the actual procedure applied within the development of so-called Creatures software, when the digital environment of acting game characters represents a type of (simplified) catalogue of "laws of nature". Obviously, this catalogue is nothing but a list of program lines. Cf. [61]; For the procedural space see in particular ibid., p. 82. For technical details concerning interstellar matter see [62].

See in this present special Issue of "Philosophies".

See in particular [67].

A promising alternative would be a structure that can be expressed in terms of a network of one or the other kind. See [68]. I thank one of the referees for suggesting to add this short summary to the conclusion.

\section{References}

1. Kauffman, S. Investigations; Oxford University Press: Oxford, UK, 2000.

2. Zimmermann, R.E. Metaphysics of Emergence; Part 1: On the Foundations of Systems; xenomoi/momo: Berlin, Germany, 2015.

3. Sandkühler, H.J. Onto-Epistemologie. In Europaeische Enzyklopaedie zu Philosophie und Wissenschaften; Sandkühler, H.J., Ed.; Meiner: Hamburg, Germany, 1990; Volume 3, pp. 608-615.

4. Zimmermann, R.E. Nothingness as Ground and Nothing But Ground; Schellings Philosophy of Nature Revisited; xenomoi/momo: Berlin, Germany, 2014.

5. Schelling, F.W.J. Grundlegung der Positiven Philosophie; Fuhrmans, B.d.E., Ed.; [Munich Lecture Notes, 1832/33]; Bottega d'Erasmo, Torino: Turin, Italy, 1972.

6. Zeilinger, D. Doctrine of the Ciphers. Intercursions Among Zeropoint-Utopia-Core. Philosophies 2021, 6, 44. [CrossRef]

7. Zimmermann, R.E.; Hofkirchner, W. The Topos of virtuality. Part 1: From the Neuman-Nave Topos to the explication of conceptual social space. TripleC [ancient series] 2009, 7, 74-87. [CrossRef]

8. Zimmermann, R.E. Topoi of systems. On the onto-epistemic foundations of matter and information. In Information Studies and the Quest for Transdisciplinarity; Burgin, M., Hofkirchner, W., Eds.; World Scientific: Singapore, 2017; pp. 191-214. 
9. Zimmermann, R.E.; Díaz Nafría, J.M. Emergence and evolution of meaning: The GDI revisiting programme. Part 1: The progressive perspective: Top-down. Information 2012, 3, 472-503. [CrossRef]

10. Zimmermann, R.E. Matter and information as attributes of substance. Eur. Phys. J. Spec. Top. 2017, 226, 177-180. [CrossRef]

11. Penrose, R. Angular momentum. An approach to combinatorial space-time. In Quantum Theory and Beyond; Bastin, T., Ed.; Cambridge University Press: Cambridge, UK, 1971; pp. 151-180.

12. Penrose, R. Twistor theory, its aims ad achievements. In Quantum Gravity; An Oxford Symposion; Isham, C.J., Penrose, R., Sciama, D.W., Eds.; Clarendon Press: Oxford, UK, 1975; pp. 268-407.

13. Zimmermann, R.E. A Conceptual View onto the Physical Foundations of Astrobiology. In Provisional Outline of a Research Program; Leibniz Online: Berlin, Germany, 2020; Issue 39, pp. 1-28.

14. Herfray, Y. New Avenues for Einstein's Gravity. From Penrose's Twistors to Hitchin's Three-Forms. Ph.D. Thesis, l'Université de Lyon, Lyon, France, 2017. Available online: https:/ / tel.archives-ouvertes.fr/tel-01735039v3 (accessed on 12 March 2021).

15. Ward, R.S.; Wells, R.O., Jr. Twistor Geometry and Field Theory; Cambridge University Press: Cambridge, UK, 1990.

16. Atiyah, M.; Dunajski, M.; Mason, L.J. Twistor theory at fifty. From contour integrals to twistor strings. Proc. R. Soc. A 2017, 473, 20170530. [CrossRef] [PubMed]

17. Crutchfield, J.P. The calculi of emergence. computation, dynamics, and induction. Physica D 1994, 75, 11-54. [CrossRef]

18. Smith, E.; Morowitz, H.J. The Origin and Nature of Life on Earth; The Emergence of the Fourth Geosphere; Cambridge University Press: Cambridge, UK, 2016.

19. Eigen, M.; Schuster, P. The hypercycle. A principle of natural self-organization Part A: Emergence of the hypercycle. Naturwissenschaften 1977, 64, 541-565. [CrossRef] [PubMed]

20. Eigen, M.; Schuster, P. The hypercycle. A principle of natural self-organization Part B: The abstract hypercycle. Naturwissenschaften 1978, 65, 7-41. [CrossRef]

21. Van de Vijver, G.; Salthe, S.N.; Delpos, M. (Eds.) Evolutionary Systems; Biological and Epistemological Perspectives on Selection and Self-Organization; Kluwer: Dordrecht, The Netherlands, 1998.

22. Penrose, R.; Rindler, W. Spinors and Space-Time; Cambridge University Press: Cambridge, UK, 1984; Volume 2.

23. Hughston, L.P. Twistors and Particles; Springer: Berlin/Heidelberg, Germany, 1979.

24. Huggett, S.A.; Tod, K.P. An Introduction to Twistor Theory; Cambridge University Press: Cambridge, UK, 1985.

25. Penrose, R. Palatial twistor theory and the twistor googly problem. Phil. Trans. R. Soc. A 2015, 373, 1-14. [CrossRef] [PubMed]

26. Penrose, R. Towards an objective physics of bell nonlocality. Palatial twistor theory. In Quantum Nonlocality and Reality; 50 Years of Bell's Theorem; Bell, M., Gao, S., Eds.; Cambridge University Press: Cambridge, UK, 2016; pp. 400-418.

27. Zimmermann, R.E. Selbstreferenz und Poetische Praxis; [Self-Reference and Poetical Praxis]; Junghans: Cuxhaven, Germany, 1991.

28. Zimmermann, R.E. Initiale Emergenz und kosmische Evolution. Zur Rekonstruktion der Substanz-Metaphysik. System Struktur 1993, I, 39-55.

29. Zimmermann, R.E. Rem Gerere. Syst. Struktur 1998, VI, 149-228.

30. Penrose, R. Afterword. In The Geometric Universe; Huggett, S.A., Mason, L.J., Tod, K.P., Tsou, S.T., Woodhouse, N.M.J., Eds.; Science, Geometry, and the Work of Roger Penrose; Oxford University Press: Oxford, UK, 1998; pp. $423-431$.

31. Nawarajan, D. Complex Spacetimes and the Newman-Janis Trick. Master's Thesis, School of Mathematics and Statistics, Victoria University of Wellington, Wellington, New Zealand, 2015.

32. Flaherty, E.J. Hermitian and Kaehlerian Geometry in Relativity; Springer: Berlin/Heidelberg, Germany, 1976.

33. Zimmermann, R.E. System des Transzendentalen Materialismus; Mentis: Paderborn, Germany, 2004; p. 437.

34. Schlosshauer, M. Decoherence and the Quantum-to-Classical Transition; Springer: Berlin/Heidelberg, Germany, 2007.

35. Szabó, G.; Fáth, G. Evolutionary Games on Graphs. Available online: www.arxiv.org/pdf/cond-mat/0607344 (accessed on 23 February 2021).

36. Loll, R. The Loop formulation of gauge theory and gravity. In Knots and Quantum Gravity; Baez, J., Ed.; Clarendon Press: Oxford, UK, 1994; pp. 1-19.

37. Rovelli, C. Loop quantum gravity. Living Rev. Relativ. 2008, 11, 1-69. Available online: https:/ /link.springer.com/article/10.129 42/1rr-2008-5 (accessed on 23 February 2021). [CrossRef] [PubMed]

38. Rovelli, C. Quantum Gravity; Cambridge University Press: Cambridge, UK, 2004.

39. Kauffman, L.H. Knots. In Geometries in Nature, Living Systems, and Human Cognition; Boi, L., Ed.; World Scientific: Singapore, 2005; pp. 131-202.

40. Zimmermann, R.E. Topological aspects of biosemiotics. TripleC 2007, 5, 49-63. [CrossRef]

41. Zimmermann, R.E. Laudatio for Louis H. Kauffman, Bertalanffy prize 2016, Freud University, Vienna. In Ontologische Probleme der Grundlegung nach Schelling und Bloch; Zimmermann, R.E., Ed.; Kovač: Hamburg, Germany, 2018; pp. $189-197$.

42. Barrett, J.W.; Crane, L. Relativistic spin networks and quantum gravity. J. Math. Phys. 1998, 39, 3296-3302. [CrossRef]

43. Crane, L.; Perez, A.; Rovelli, C. Perturbative finiteness in spin-foam quantum gravity. Phys. Rev. Lett. 2001, 87, 181301. [CrossRef]

44. Baez, J.; Christensen, D. Spin Foams and Gauge Theories. E-mail Discussion. 2000. Available online: http://jdc.math.uwo.ca/ spin-foams /s.p.r (accessed on 23 February 2021).

45. Baez, J. Higher-dimensional algebra and planck scale physics. In Physics Meets Philosophy at the Planck Scale; Callender, C., Huggett, N., Eds.; Cambridge University Press: Cambridge, UK, 2001; pp. 177-196. 
46. Zimmermann, R.E. The foundation of the world. Conceptual notes on procedural space. In Proceedings of the 69th Annual Meeting of the German Physical Society, University of Technology, Berlin, Germany, 4-9 March 2005; akphil 2.3, Proceedings. Volume 5, p. 44.

47. Kunihiko, K. Chaos as a source of complexity and diversity in evolution. In Artificial Life; MIT Press: Cambridge, MA, USA, 1995; pp. 163-177.

48. Newman, M.E.J. The structure and function of complex networks. SIAM Rev. 2003, 45, 167-256. [CrossRef]

49. Dovogartsev, S.N.; Mendes, J.F.F. Evolution of Networks; Oxford University Press: Oxford, UK, 2003.

50. Davidsen, J.; Ebel, H.; Bornholdt, S. Emergence of a Small World from Local Interactions: Modeling Acquaintance Networks. 2001. Available online: www.arxiv.org/pdf/cond-mat/0108302 (accessed on 24 June 2021).

51. Pokornyi, Y.V.; Borovskikh, A.V. Differential equations on networks (geometric graphs). J. Math. Sci. 2004, 119, 691-717. [CrossRef]

52. Ruthotto, L.; Haber, E. Deep Neural Networks Motivated by Partial Differential Equations. 2018. Available online: www.arxiv. org/pdf/1804.04272 (accessed on 23 February 2021).

53. Chen, R.T.Q.; Rubanova, Y.; Bettencourt, J.; Duvenaud, D. Neural Ordinary Differential Equations. 2019. Available online: www.arxiv.org/pdf/1806.07366 (accessed on 23 February 2021).

54. Wu, Z.; Menichetti, G.; Rahmede, C.; Bianconi, G. Emergent Complex Network Geometry. 2015. Available online: www.arxiv. org/pdf/1412.3405 (accessed on 23 February 2021).

55. Jain, S.; Krishna, S. Graph theory and the evolution of autocatalytic networks. In Handbook of Graphs and Networks; Bornholdt, S., Schuster, H.-G., Eds.; Wiley-VCH: Weinheim, Germany, 2003; pp. 355-395.

56. Kauffman, S.A. A World Beyond Physics; Oxford University Press: Oxford, UK, 2019.

57. Montévil, M.; Mossio, M. Biological organization as closure of constraints. J. Theor. Biol. 2015, 372, 179-191. [CrossRef] [PubMed]

58. Farmer, J.D.; Kauffman, S.A.; Packard, N.H. Autocatalytic replication of polymers. Physica D 1986, 22, 50-67. [CrossRef]

59. Harvey, G. Animism: Respecting the Living World; Columbia University Press: New York, NY, USA, 2006.

60. Smolin, L. The Life of the Cosmos; Oxford University Press: Oxford, UK, 1997.

61. Grand, S. Creation. Life and How to Make It; Harvard University Press: Cambridge, MA, USA, 2001.

62. Dyson, J.E.; Williams, D.A. The Physics of Interstellar Medium, 2nd ed.; IOP Publishing: Bristol, PA, USA, 1997.

63. Nascimento Vieira, A.d.; Nagies, F.S.P.; Martin, W.F. To what inanimate matter are we most closely related, and does the origin of life harbor meaning? Philosophies 2021, 6, 33.

64. Schulze-Makuch, D.; Irwin, L.N. Life in the Universe, 3rd ed.; Expectations and Constraints; Springer: Berlin/Heidelberg, Germany, 2018.

65. Zimmermann, R.E. Systems of Systems as Represented by Categories; Hofkirchner, W., Kreowski, H.-J., Zimmermann, R.E., Eds.; Workshop Systems of Systems emcsr2016, Vienna, Book of Abstracts; Available online: https:/ / emcsr.net/ (accessed on 24 June 2021).

66. Penrose, R.; Hameroff, S.; Kak, S. (Eds.) Consciousness and the Universe: Quantum Physics, Evolution, Brain E Mind; Cosmology Science Publishers: Cambridge, MA, USA, 2011.

67. Penrose, R.; Hameroff, S. Consciousness in the Universe. Neuroscience, Quantum Space-Time Geometry and Orch OR Theory; Penrose, R., Hameroff, S., Kak, S., Eds.; Cosmology Science Publishers: Cambridge, MA, USA, 2011; pp. 8-47.

68. Ilachinski, A. Cellular Automata; World Scientific: Singapore, 2001.

69. Mitchell, E.D.; Staretz, R. The Quantum Hologram and the Nature of Consciousness; Penrose, R., Hameroff, S., Kak, S., Eds.; Cosmology Science Publishers: Cambridge, MA, USA, 2011; pp. 82-114.

70. Baez, J.C.; Biamonte, J.D. A Course on Quantum Techniques for Stochastic Mechanics. 2012. Available online: www.arxiv.org/ pdf/1209.3632 (accessed on 23 February 2021).

71. Wittgenstein, L. Philosophische Untersuchungen, 9th ed.; Suhrkamp: Frankfurt, Germany, 2003.

72. Lloyd, S. Programming the Universe; Vintage/Random House: New York, NY, USA, 2006.

73. Gleiser, M. The three origins. Cosmos, life, and mind. In Science and Ultimate Reality; Barrow, J.D., Davies, P.C.W., Harper, C.L., Jr., Eds.; Cambridge University Press: Cambridge, UK, 2004; pp. 637-653. 